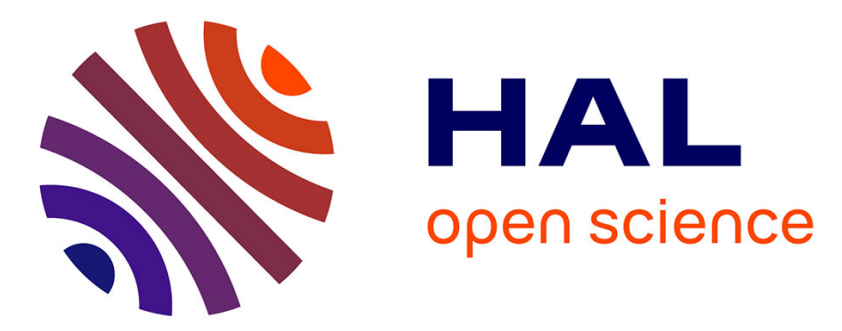

\title{
Isostructural phase transition by point defect reorganization in the binary type-I clathrate Ba7.5Si45
}

Régis Debord, Holger Euchner, Vittoria Pischedda, Michael Hanfland, Alfonso San-Miguel, Patrice Mélinon, Stéphane Pailhès, Denis Machon

\section{- To cite this version:}

Régis Debord, Holger Euchner, Vittoria Pischedda, Michael Hanfland, Alfonso San-Miguel, et al.. Isostructural phase transition by point defect reorganization in the binary type-I clathrate Ba7.5Si45. Acta Materialia, 2021, 210, pp.116824. 10.1016/j.actamat.2021.116824 . hal-03227682

\section{HAL Id: hal-03227682 \\ https://hal.science/hal-03227682}

Submitted on 17 May 2021

HAL is a multi-disciplinary open access archive for the deposit and dissemination of scientific research documents, whether they are published or not. The documents may come from teaching and research institutions in France or abroad, or from public or private research centers.
L'archive ouverte pluridisciplinaire HAL, est destinée au dépôt et à la diffusion de documents scientifiques de niveau recherche, publiés ou non, émanant des établissements d'enseignement et de recherche français ou étrangers, des laboratoires publics ou privés. 


\title{
Isostructural phase transition by point defect reorganization in the binary type-I clathrate $\mathrm{Ba}_{7.5} \mathrm{Si}_{45}$
}

\author{
Régis Debord ${ }^{1}$, Holger Euchner ${ }^{2}$, Vittoria Pischedda ${ }^{1}$, Michael Hanfland ${ }^{3}$, Alfonso \\ San-Miguel ${ }^{1}$, Patrice Mélinon ${ }^{1}$, Stéphane Pailhès ${ }^{1}$, Denis Machon ${ }^{1}$
}

1 - Institut Lumière Matière, Université de Lyon, Université Claude Bernard Lyon 1, CNRS UMR 5306, 69622 Villeurbanne, France

2 -Helmholtz Institute Ulm for Electrochemical Energy Storage (HIU), Helmholtzstr. 11, 89081 Ulm, Germany

3 - European Synchrotron Radiation Facility, BP 220, 38043, Grenoble Cedex, France

Corresponding authors: denis.machon@univ-lyon1.fr ; stephane.pailhes@univ-lyon1.fr

\begin{abstract}
Competition between point defect (vacancy and interstitial) microscopic configurations is inherent to crystalline phases of increased structural complexity. Phase transitions that preserve symmetry between them belong to a specific class of isostructural transitions. Type-I silicon clathrates are complex crystalline phases whose unit cell containing fifty atoms consists of a 3D covalent silicon framework of face-sharing polyhedral cages encapsulating guest cations. At high pressure, an intriguing structural transition is associated with an abrupt reduction of volume with no indication for any breakage of symmetry. Using isothermal high-pressure Xray diffraction performed on a single crystal of the simplest representative type-I silicon clathrates, binary $\mathrm{Ba}_{8} \mathrm{Si}_{46}$, we confirm the isostructural character of the transition and identify the associated mechanism. A detailed analysis of the atomic structural parameters across the transition in combination with ab initio studies allow us to pinpoint a microscopic mechanism driven by a rearrangement of point defects initially present in the structure. An analysis based on the Landau theory gives a coherent description of the experimental observations. A discussion on the analogy between this transformation and liquid-liquid transitions is proposed.
\end{abstract}




\section{Introduction}

Exploiting the structural complexity of crystalline materials is a powerful strategy for designing novel materials [1]. For instance, it allows disentangling electronic and heat transport which is of significant interest in the field of thermoelectricity $[2,3,4,5]$. The existence of a rich defect landscape is inherent to complex structural phases in which energetically degenerate defect configurations compete. Their relative thermodynamic stability is determined by the structure and stoichiometry of the crystal unit cell, and/or the thermodynamic conditions. It has been shown theoretically that a first-order isostructural transition between two defect configurations may occur as long as there is no ordering of defects [6]. This first-order disorder-disorder transition in crystals exhibits a transition line in the pressure-temperature phase diagram terminated by a critical point $[7,8]$. Here, we report an experiment showing such phase transitions associated with a change of the defect configuration in the simplest representative of the type-I clathrate structure, namely the binary $\mathrm{Ba}_{8} \mathrm{Si}_{46}$, whose complex guest-host cagebased structure allows improving thermoelectric efficiency, widely studied in the field of electrical-heat conversion.

Due to the lack of symmetry breaking, isostructural transitions are extremely difficult to identify while they can lead to considerable changes in material properties. Also, investigating their underlying microscopic mechanisms is challenging. The best-known cases are of electronic origin and are associated with either a modification of the hybridization between valence and conduction band states, as in some rare earth crystals [7, 9], or a change of the electron correlation in strongly correlated systems, such as in Cr-doped $\mathrm{V}_{2} \mathrm{O}_{3}$ [10]. Much rarer are isostructural transitions driven by non-electronic mechanisms. In the crystalline salt $\mathrm{SnCl}_{2}-$ $2 \mathrm{H}_{2} \mathrm{O}$, an isostructural transition was ascribed to the orientational ordering of $\mathrm{H}_{2} \mathrm{O}$ molecules trapped in the $\mathrm{SnCl}_{2}$ lattice $[11,12]$. In the cage-based $\beta$-pyrochlore oxide $\mathrm{KOs}_{2} \mathrm{O}_{6}$, an isostructural transition occurring upon cooling below $7.5 \mathrm{~K}$ was ascribed to a change of the average $\mathrm{K}$ ion positions inside the $\mathrm{OsO}_{6}$ cages [for a review see 13].

Type-I clathrates possess a complex unit cell, containing more than 50 atoms, in which point defects of different natures (vacancy, partial substitution) are used to engineer their electrical and thermal properties [14]. Their crystal structure consists of a 3D host network of mostly covalently bound cages of group 14 elements that encapsulate alkali or alkali-earth cations and are arranged in a cubic unit cell (space group $\left.\operatorname{Pm} \overline{3} n\left(\mathrm{~N}^{\circ} 223\right), \mathrm{a} \sim 1 \mathrm{~nm}\right)[14,15]$. The geometrical arrangement of the framework consists of two dodecahedral (20-atom) and six 
larger tetrakaidecahedral (24-atom) cages, as shown in Figure 1 for the binary type-I silicon clathrate $\mathrm{Ba}_{8} \mathrm{Si}_{46}$ of interest here. Upon isothermal compression at room temperature, an "homothetic volume collapse", i.e. a significant decrease of the unit cell volume without change of the crystal symmetry has been reported between 13-16 GPa in type-I silicon clathrates [16, 17, 18, 19, 20, 21, 22, 23]. Different microscopic mechanisms have been proposed, from electronic transitions [24, 25] over phonon instabilities [26] to the pressure-induced activation of Si vacancy diffusion [27]. However, up to now, no consensus has been reached.

Here, we report a diffraction study of the pressure-temperature (P-T) phase diagram in a Ba8${ }_{x} \mathrm{Si}_{46-\mathrm{y}}$ single crystal grown at high pressure and high temperature, where $\mathrm{x}$ and y quantify the guest and host vacancy contents, respectively. In agreement with previous experiments performed on polycrystalline samples, a pressure-induced isostructural transition is observed at $16.8 \mathrm{GPa}$ at $300 \mathrm{~K}$ whose slope in the (P-T) phase diagram is found to be negative. A detailed analysis of the changes of the structural parameters in combination with $a b$ initio simulations converge to a microscopic mechanism based on a pressure-induced local disorder-disorder transition. This transition is initiated by the transformation of a Ba-vacancy, present in the crystal structure at atmospheric pressure, into a vacancy in the Si-framework without overall symmetry breaking. Intriguingly, this Si-framework atom moves in the host cage network and adopts a quasi-neutral electronic configuration, resulting in the addition of a high density close to Fermi energy. The experimental observations are described within the framework of the Landau theory applied to isostructural phase transitions. 


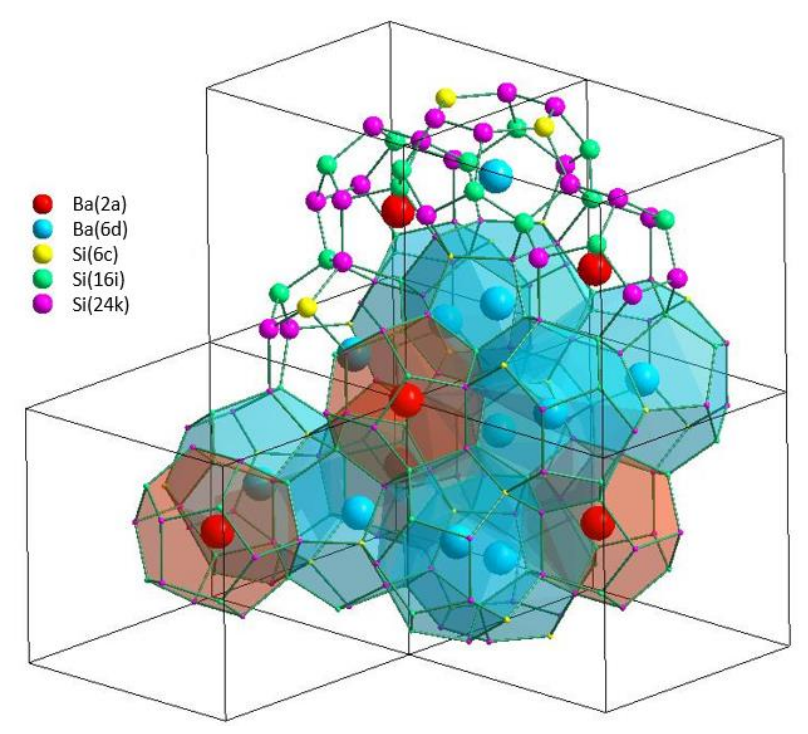

Figure 1 - Representation of the type-I silicon clathrate $\mathrm{Ba}_{8} \mathrm{Si}_{46}$ unit cell (Space group $P m \overline{3} n$ (No. 223)). Atomic positions were obtained from single crystal data refinement of the diffraction data collected in run 1 at ambient pressure and temperature. The $\mathrm{Ba}(2 \mathrm{a}), \mathrm{Ba}(6 \mathrm{~d})$, $\mathrm{Si}(6 \mathrm{c}), \mathrm{Si}(16 \mathrm{i})$ and $\mathrm{Si}(24 \mathrm{k})$ atoms are represented in red, blue, yellow, green, and purple, respectively. The solid lines of the cubes indicate the edges of the unit whose lattice constant is $10.317 \AA$ at ambient pressure and temperature conditions.

\section{Methods}

\section{Microcrystal synthesis, physical characterization}

A high-pressure high-temperature belt-type apparatus was used to synthesize clathrate singlecrystals. The first step is to compress at ambient temperature a mixture of $\mathrm{BaSi}_{2}$ (Cerac, 98\%) and $\mathrm{Si}$ (Strem, 99.999\%) powders up to $3 \mathrm{GPa}$, and then slowly heat it to $1300 \mathrm{~K}$ at a rate of 2 $\mathrm{K} / \mathrm{min}$, followed by a dwell period of $30 \mathrm{~min}$ before final quenching. Typical dimensions of the single crystal used in the present study consist of an area of about $50 \times 60 \mu \mathrm{m}^{2}$ with a thickness of $20 \mu \mathrm{m}$.

At ambient pressure and $300 \mathrm{~K}$, the $\mathrm{Ba}_{8-\mathrm{x}} \mathrm{Si}_{46-\mathrm{y}}$ structure is described by the space group $\operatorname{Pm} \overline{3} n$ (No. 223) with a cell parameter of $a\left(\mathrm{~T}=300 \mathrm{~K}, \mathrm{P}_{\mathrm{atm}}\right)=10.3176(7) \AA$ (Figure 1 and see Supplementary Materials (Tab. SM1)). The resulting composition corresponds to the extended formulae $\mathrm{Ba}_{1.5} \square \square_{0.5} \mathrm{Ba}_{6} \mathrm{Si}_{45} \square$ ( $\square$ stands for a vacancy) as the single crystal refinement reveals an amount of about $0.5 \mathrm{Ba}$ vacancies, mainly localized on the $\mathrm{Ba}(2 \mathrm{a})$ site in the small $\mathrm{Si}_{20}$ cages, while the $\mathrm{Ba}(6 \mathrm{~d})$ site in the $\mathrm{Si}_{24}$ cages was found to be fully occupied (see Tab. SM- 
2). In the following, the reduced formulae $\mathrm{Ba}_{7.5} \mathrm{Si}_{45}$ will be adopted for the sake of readability. Vacancies mostly localized on the $\mathrm{Ba}(2 \mathrm{a})$ site are commonly observed in type-I Si clathrates, independently of the synthesis method. The lattice parameter is found to decrease linearly with the $\mathrm{Ba}(2 \mathrm{a})$ vacancy content $[25,28,29,30]$. Our values of the cell parameter and $\mathrm{Ba}(2 \mathrm{a})$ vacancy content are in good agreement with this linear tendency. One Si vacancy is predominantly found on the $16 i$-site (Tab. SM-2). There is neither an indication of a superstructure in the whole data collection nor the signature of a $\mathrm{Ba}$ off-centering in the atomic displacement parameters. Thus, no correlated defect structure is associated with the $\mathrm{Ba} / \mathrm{Si}$ vacancies which can therefore be viewed as simple point defects. Moreover, our previous experimental and theoretical studies of the lattice dynamics on the same material show that it is an essentially harmonic crystal without unusually large anharmonicities [31, 32, 33].

\section{Diffraction experiment at high pressure and refinement}

High-pressure X-ray diffraction experiments were performed at the European Synchrotron Radiation Facility (ESRF) on beamline ID09 (wavelength of $0.4155 \AA$ ) using microcrystals of the type-I silicon clathrate $\mathrm{Ba}_{8-\mathrm{x}} \mathrm{Si}_{46-\mathrm{y}}$. A diamond anvil cell (DAC) was used with a stainlesssteel gasket presenting a $250 \mu \mathrm{m}$ hole at its center. The in-situ pressure was probed by the ruby fluorescence technique [34]. Datasets from three runs are presented: (Run 1) an isothermal compression at $300 \mathrm{~K}$ performed on microcrystal 1 (see Tab. SM-1); (Run 2), an isothermal compression at $180 \mathrm{~K}$ on microcrystal 2 (see Tab. SM-3). (Run 3) an isothermal compression at $450 \mathrm{~K}$ performed on a powder sample. Note that only data recorded in Run 1, for which the high quality of our single crystal was preserved over the entire pressure range of the experiment, were used for precise refinements of the microscopic structural parameters, providing us with the new insights on which the present discussion on the transition mechanism is based. Moreover, data collected in runs 2 and 3 were used for determining the pressure dependence of the cubic unit cell parameter and the transition pressure.

In runs 1 and 2 , the microcrystal was loaded in the DAC filled with $\mathrm{He}$ as the pressure transmitting medium (PTM). In run 2, the DAC with the sample was inserted in a He-cryostat. In run 3, the powder sample was loaded in the DAC filled with Neon. Neon was used as PTM in order to prevent the insertion of $\mathrm{He}$ in the crystalline structure at high temperature. The whole device was inserted in a heating ring.

In runs 1 and 2, more than 30 diffraction collections were recorded between ambient pressure and $25 \mathrm{GPa}$. Each collection contained 120 images, corresponding to an angular scan in the range allowed by the DAC optical aperture (scan between $-30^{\circ}$ and $+30^{\circ}$ with an angular step 
of $0.5^{\circ}$ ). More than 2000 peak locations were observed per collection (i.e., each pressure increment), which corresponds to 700 independent reflections. The analysis and refinement of the data were performed using Crysalis and Jana2006 software [35]. Regarding the refinement of high-pressure data, we performed a two-step process with Jana2006 to disentangle the site occupancy factors (SOF) and the isotropic atomic displacement parameters $\left(\mathrm{U}_{\mathrm{iso}}\right)$. First, the SOFs were refined on a small scale, $0<\sin (\theta)<0.5$, since the atomic scattering factor strongly decreases with $\sin (\theta)$. In the second step, SOFs were fixed and other parameters $\left(U_{\text {iso }}\right.$, Wyckoff positions) were refined.

\section{Ab initio simulations}

Total energy calculations on the perfectly ordered composition $\mathrm{Ba}_{8} \mathrm{Si}_{46}$ were conducted, using the periodic density functional theory (DFT) code VASP [36]. VASP is a plane wave code in which the electron-ion interaction is described by pseudopotentials. For our study, we applied the projector augmented wave method [37], while the exchange and correlation were accounted for by the generalized gradient approximation in the formulation of Perdew and Wang [38]. A $5 \times 5 \times 5 \mathrm{k}$-point mesh and an energy cutoff of $500 \mathrm{eV}$ were applied for all the calculations. The pressure was set within the VASP code (PSTRESS tag), which allows to choose an external pressure. The implementation in VASP is such that the given pressure is added to the stress tensor. Phonon calculations were carried out by using the PHONON code [39]. The finite displacement method was applied for the determination of the harmonic force constants, necessary to obtain the dynamical matrix.

\section{Results and Discussion}

The normalized pressure dependence of the unit cell volume, $\mathrm{V}(\mathrm{P}) / \mathrm{V}_{0}$, is shown for the three runs in Fig. 2a-c. For the compression at $300 \mathrm{~K}$ (Run 1), a discontinuity in the $\mathrm{V}(\mathrm{P}) / \mathrm{V}_{0}$ curve is observed at $16.8 \pm 0.3 \mathrm{GPa}$. This corresponds to a well-known phase transition, reported in several polycrystalline samples of type-I silicon clathrates [21]. In the following, we assume that this transition separates a low-pressure phase, called "Phase I", from a high-pressure phase, "Phase II". A similar transition was also observed for the compression at $180 \mathrm{~K}$ (Run 2) but at a higher pressure of $21 \pm 0.3 \mathrm{GPa}$, and with a larger amplitude. Apparently, no discontinuity in $\mathrm{V}(\mathrm{P}) / \mathrm{V}_{0}$ was resolved on the compression at $450 \mathrm{~K}$ (Run 3), a difference of behavior which will be more evident in the $F-f$ plot analysis performed below. The respective onsets of the phase transition are summarized in the pressure-temperature (P-T) phase diagram in Fig. $2 d$. 

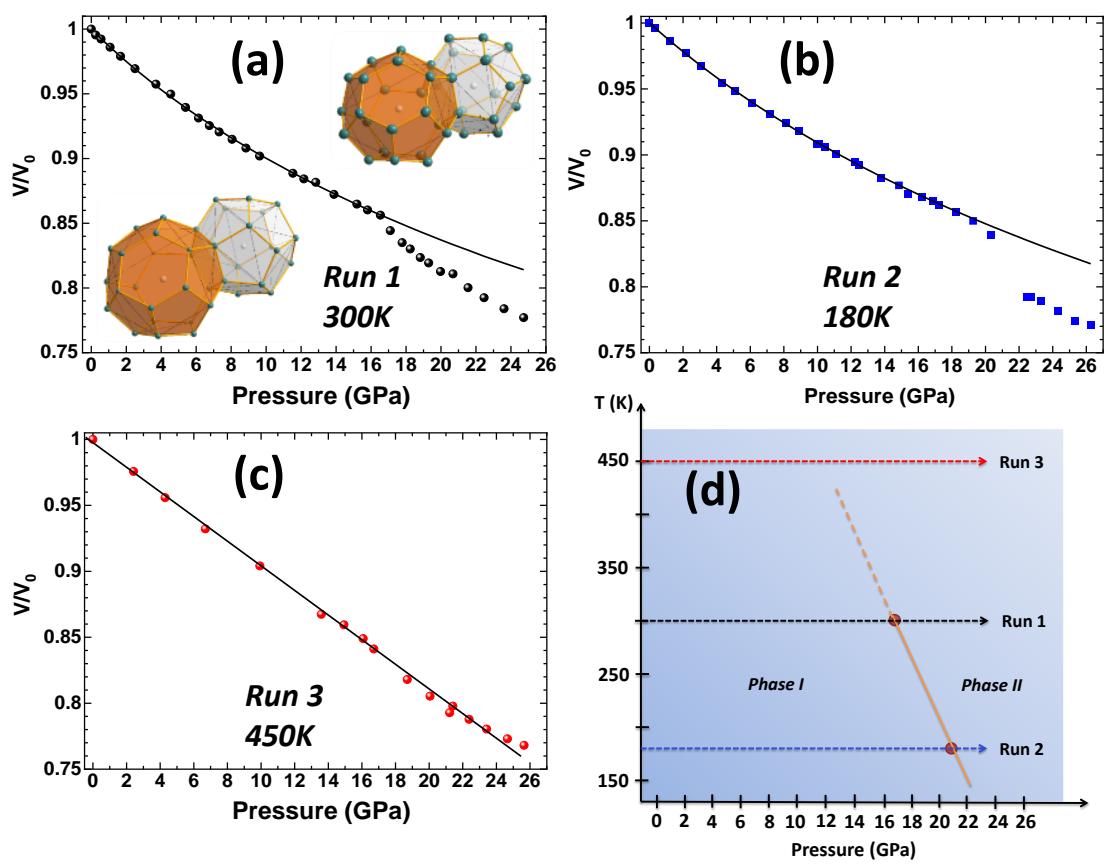

Figure 2 - Pressure dependence of the unit cell volume of $\mathrm{Ba}_{1.5} \square_{0.5} \mathrm{Ba}_{6} \mathrm{Si}_{45} \square_{1}$ (reduced formulae: Ba7.5 $\mathrm{Si}_{45}$ ) normalized to its value at $\mathrm{P}_{\mathrm{atm}}$ at (a) $300 \mathrm{~K}$, (b) $180 \mathrm{~K}$ and (c) $450 \mathrm{~K}$. In the insets of (a), the cage building units, $\mathrm{Si}_{24}$ (yellow) and $\mathrm{Si}_{20}$ (white), at $\mathrm{P}_{\text {atm }}$ (bottom) and $23.6 \mathrm{GPa}$ (top), above the pressure transition, obtained from refinements of data collected in run 1; (d) PressureTemperature phase diagram of $\mathrm{Ba}_{7.5} \mathrm{Si}_{45}$, showing the isostructural transition line (yellow solid line) and the isothermal compression paths followed in runs 1-3. In the temperature between room temperature (run 1) and $450 \mathrm{~K}$, the isostructural is represented by a dotted line indicating the existence of a critical point somewhere in this temperature and pressure range

No symmetry breaking is observed in the diffraction maps when going from phase I to phase II. The Bragg spots are distorted under pressure, which is mainly due to pressure-induced strain effects at the transition, but they remain mostly homogeneous and do not split. An example of diffracted intensity maps around the high symmetry Bragg peak (330) and the Bragg peak (211) are shown for different pressure values on both sides of the transition (Figure 3). The structure factor of peak (221) is dominated by the guest atom at site $2 a$ which, as we will see below, plays an essential role in the transition. Below the transition pressure no or only slight deviations from the spherical peak shape are observed. Above the transition, the peak shape becomes elliptic, without evidence of splitting or any presence of satellite spots. 


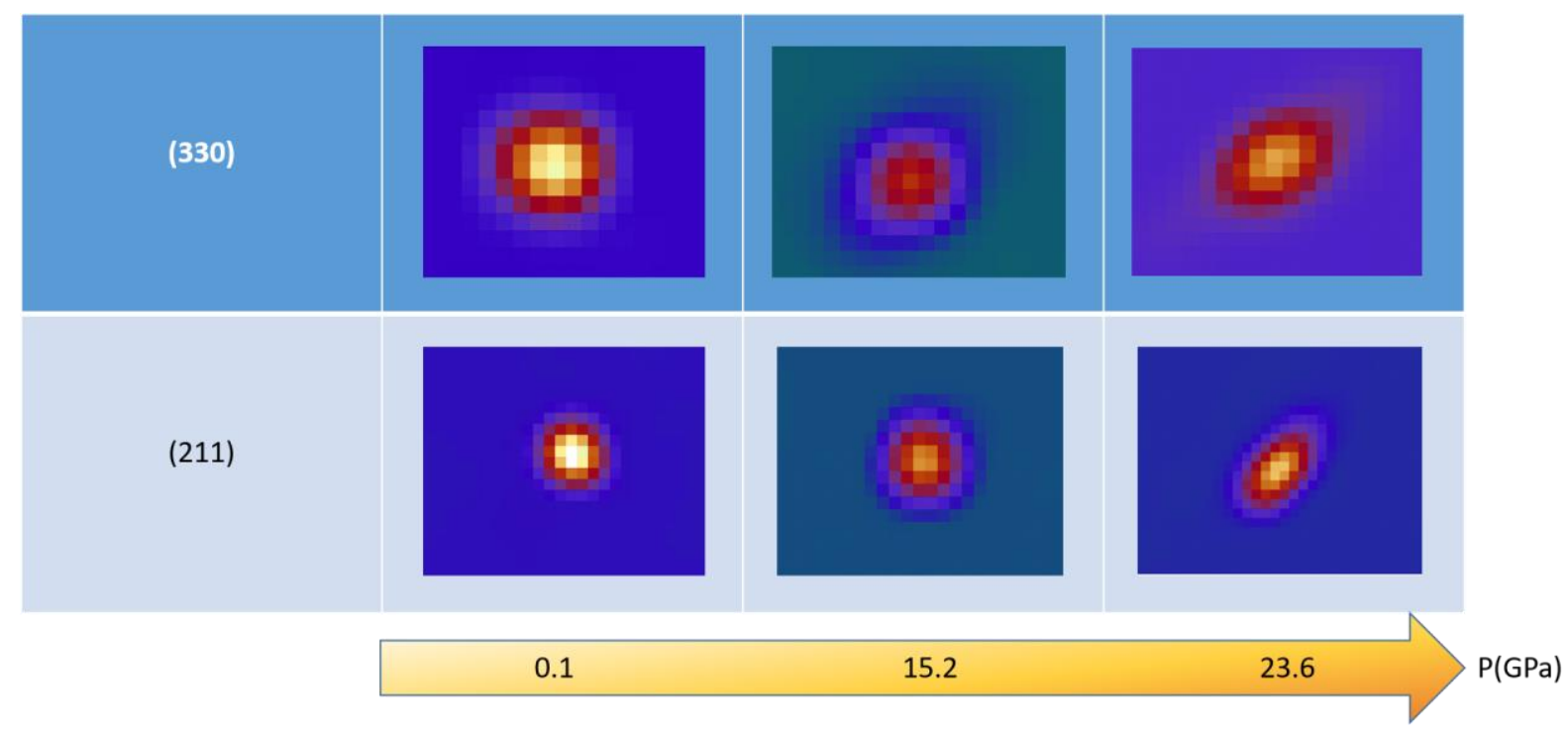

Figure 3 - Diffracted intensity maps around the high symmetry Bragg peak (330) and (211) at three different pressures during Run 1 on both sides of the iso-structural transition at $\mathrm{P}=16.8$ $\mathrm{GPa}$.

As a result, the dataset of phase II is also best refined within the space group $\operatorname{Pm} \overline{3} n\left(\mathrm{~N}^{\circ} 223\right)$. The refined cage structures for both phases are represented in Fig. 2a. The volume ratio of the $\mathrm{Si}_{20}$ and $\mathbf{S i}_{24}$ cages, determined experimentally (see Tab $\mathbf{S M - 4}$ ), is constant in the whole pressure range, confirming that the abrupt volume contraction at the transition is homothetic. The preservation of the space group across the transition is a strong indication of the isostructural character of the transition from phase I to phase II. The $\mathrm{V} / \mathrm{V}_{0}$ data for $\mathrm{Ba}_{7.5} \mathrm{Si}_{45}$ upon compression at ambient temperature (Run 1) in the low-pressure range 0.1-17 GPa were initially fitted using a third-order Birch-Murnaghan equation of state model to obtain the bulk modulus $\mathrm{K}_{0}$ and its pressure derivative $\mathrm{K}_{0}$ ' for the clathrate phase. The values obtained by this procedure were $\mathrm{K}_{0}=75(1) \mathrm{GPa}$ and $\mathrm{K}_{0}{ }^{\prime}=4.2(2)$. Those values are in good agreement with $a b$ initio calculations [23], and slightly lower than previous experimental studies [21]. Moreover, the value of $\mathrm{K}_{0}$, obtained at $300 \mathrm{~K}$ in the low-pressure range, agrees with the bulk modulus, $K_{0}=\frac{C_{11}+2 C_{12}}{3}=79 \mathrm{GPa}$ that can be extracted from measurements of the elastic constants $\left(\mathrm{C}_{11}\right.$ and $\mathrm{C}_{12}$ ) by inelastic $\mathrm{X}$-Ray scattering under normal atmospheric pressure and at $300 \mathrm{~K}$ [32] and with the calculated value obtained by first-principles calculation based density-functional theory [40]. 
However, since $\mathrm{V}(\mathrm{P})$ is a nonlinear relation, designing a goodness of fit criterion and choosing the best set of parameters can be difficult. The alternative fitting procedure used here involves the transformation of the $\mathrm{V}(\mathrm{P})$ data into the normalized or reduced stress-strain variables $F$ and $f$, allowing the removal of the correlations between the fitting parameters, and defined as [41]

$$
\begin{gathered}
f=\frac{1}{2}\left[\left(\frac{V_{0}}{V}\right)^{2 / 3}-1\right] \\
F=P\left[3 f(1+2 f)^{5 / 2}\right]^{-1}
\end{gathered}
$$

This formalism yields the second-order finite strain equation

$$
F=K_{0}\left[1-\frac{3}{2}\left(4-K^{\prime}\right) f\right]
$$

We can then conduct a linear fit to the resulting $F-f$ plot. In this case, well accepted goodness of fit criteria can be applied to the linear fit and likely $v s$ unlikely ranges of values for $K_{0}$ and $K^{\prime}$ can be readily tested against the data within its error bars. $K_{0}$ and $K^{\prime}$ were then obtained as the intercept $f \rightarrow 0$ and the slope of the $F$ - $f$ plot, respectively. $F$ - $f$ plots for the three experiments are shown in Fig. 4.

The $F-f$ plot is marked by a step-like change whose edge appears at the transition pressure for runs 1 and 2. On the other hand, the $F$-f plot associated with run 3 shows a continuous evolution of elastic properties over the whole pressure range. The question that arises is whether or not the clathrate structure has undergone the I-to-II transformation at ambient pressure upon heating from $300 \mathrm{~K}$ to $450 \mathrm{~K}$. This has not been seen in previous high temperature studies [42] and, furthermore, it would deviate significantly from the P-T transition line whose extrapolation at $\mathrm{P}_{\mathrm{atm}}$ gives a transition temperature of about $780 \mathrm{~K}$ (see Fig. 2d). In addition, the increase of the unit cell volume upon heating from $180 \mathrm{~K}$ to $450 \mathrm{~K}$ at $\mathrm{P}_{\mathrm{atm}}$ is fully reproduced by the effect of the lattice thermal expansion $\alpha=1.210^{-5} \mathrm{~K}^{-1}$ in $\mathrm{Ba}_{8} \mathrm{Si}_{46}$ [43]. All this confirms the absence of a transition in run 3 and a continuous transformation of phase I to phase II. This behaviour suggests the existence of a critical point ending the isostructural transition line in the range [300:480 K], as in the case of the liquid-gas transformation.

Note that close examination of the $F$ - $f$ curve for run 1 shows a complex behavior with two different elastic regimes in the [0-17 GPa] range. An initial monotonous regime can be seen from 0.1 to $\sim 5 \mathrm{GPa}$, followed by a sudden softening and a new monotonous regime from $\sim 5$ to 
$\sim 17 \mathrm{GPa}$. The mechanical properties for both regimes are reported in Table I. The values of $\mathrm{K}_{0}$ ' are quite high compared to previous studies that did not use an $F-f$ plot analysis but is in quite good agreement with a study on $\mathrm{Rb}_{6.15} \mathrm{Si}_{46}$ using a similar data analysis [23]. Therefore, fitting the experimental equation of state (EOS) of clathrates should be done with great care. Using a Birch-Murnahgan EOS on the whole pressure range before the phase transition may give biased parameters. A detailed analysis based on the $F-f$ plot must be undertaken in view to extracting reliable elastic properties in the different pressure regimes.

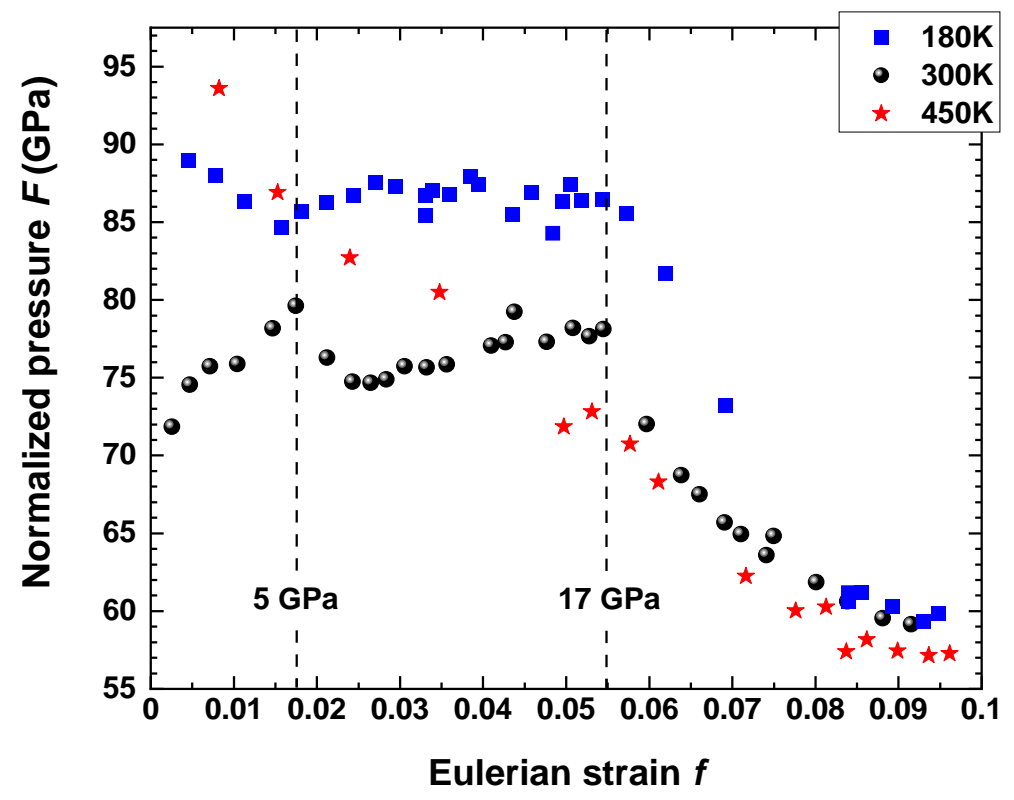

Figure 4 - Normalized pressure versus Eulerian strain $(F-f$ plot $)$ computed from the pressure dependence of the unit cell volume obtained in the isothermal compression paths followed in runs 1-3 at $180 \mathrm{~K}$ (filled blue squares), $300 \mathrm{~K}$ (filled black circles) and $450 \mathrm{~K}$ (filled red stars).

The $F$ - $f$ plot for run 2 does not show an abrupt variation until the phase transition at P $21 \mathrm{GPa}$. The refined mechanical parameters are reported in Table I. The increase of the bulk modulus value as the temperature decreases is generally observed and explained on the basis of thermodynamic considerations [44].

The preservation of the space group across the transition and the possible existence of a critical point confirm the isostructural character of the transition from phase I to phase II. As shown in Fig. 2d, the slope of the transition pressure from phase I to II, $\frac{d P_{I-I I}}{d T}$, is negative, which, through the Clapeyron relation, guarantees that phase II has a higher entropy than that of phase I. 
Table I - Mechanical properties obtained by a F-f analysis for run 1 and run 2

\begin{tabular}{cccc}
\hline \hline & & $\mathrm{K}_{0}$ & $\mathrm{~K}_{0}{ }^{\prime}$ \\
\hline \multirow{3}{*}{ Run 1 } & {$[0.1-5 \mathrm{GPa}]$} & $71.6(7)$ & $8.2(2)$ \\
& {$[5-17 \mathrm{GPa}]$} & $71.6(8)$ & $5.2(2)$ \\
& {$[0.1-17 \mathrm{GPa}]$} & $75(1)$ & $4.6(3)$ \\
\hline Run 2 & {$[0.1-20 \mathrm{GPa}]$} & $87(1)$ & $3.8(2)$ \\
\hline
\end{tabular}

The next step is to identify the microscopic order parameter of the transition. To this end, the structural parameters in phases I and II, refined within the space group $P m \overline{3} n$ (No. 223) from the data recorded in run 1 , have been studied as a function of pressure.

Two structural models are tested on the dataset of run 1, describing different possible microscopic scenarios of the isostructural transition. In the first one, proposed by litaka, the phase transition is driven by the creation of $\mathrm{Si}$ vacancies under pressure, followed by the diffusion of $\mathrm{Si}$ atoms and the segregation of a $\mathrm{Si}$-rich phase at the grain boundaries [27]. The system is assumed to be thermodynamically "open", meaning a variable number of vacancies. First principle simulations showed that the enthalpy gain of the vacancy formation at high pressure over-compensates the energy barriers between the Si sites, allowing the thermal diffusion of Si over large distances. To test this hypothesis on our data, a structural model with no constraints on the Si content was applied, i.e. the amount of vacancies was obtained directly from the refinement of the site occupancy factors (SOF). In the second scenario, the host Si atoms diffused over shorter lengths only by hopping into the unoccupied $\mathrm{Ba}(2 a)$ sites and the Si vacancies initially present in the structure, i.e. either on the host $16 i$-site or the $24 k$-site. In this case, the Si content was preserved, there was no secondary phase and the system was "closed".

In the context of the first scenario, the pressure dependence of the Si vacancy content, summed over the three $\mathrm{Si}(16 i, 24 k$ and $6 c)$ sites, is reported in Fig. 6a (individual SOFs are shown in Fig. SM-1). A sudden increase occurs at the isostructural transition. At least three additional Si vacancies, mostly found on the $6 c$ - and $24 k$-sites, appear in phase II in good agreement with the prediction of litaka [27]. However, this raises a question: where do the escaped Si atoms diffuse? Experimentally, no additional diffraction intensity due to a secondary bulk Si-rich phase was observed, in agreement with other studies [19, 30]. Moreover, we found that the additional $\mathrm{Si}$ vacancies in phase II were refilled by bulk $\mathrm{Si}$ atoms when crossing back the 
transition line upon decompression (see Fig. 6a). This reversibility questions the existence of a long-range diffusion mechanism.

In the context of the second scenario, the pressure dependences of the Si SOFs at the $\mathrm{Ba}(2 a)$ and $\operatorname{Si}(16 i, 24 k$ and $6 c)$ sites are shown in Fig. $\mathbf{6 b}$. The filling of the vacant $2 a$ sites starts at 7 GPa but without further variation until the transition pressure. This first step may induce the variation in the elastic behavior, as observed in the $F-f$ plot. An additional Raman signature of this pre-transitional effect was reported by Kume et al. [18]. Above the transition pressure, there is a clear increase of the $\mathrm{Si} \mathrm{SOF}$ on the guest $2 a$-site which is filled by $\mathrm{Si}$ atoms coming from the $24 k$ and $6 c$ sites. At the highest pressure, the latter reaches a value of 0.25 , meaning that all the initial $\mathrm{Ba}(2 \mathrm{a})$ vacancies are filled by $\mathrm{Si}$. The $\mathrm{Si}(24 k$ and $6 c)$ atoms also diffuse on the free $16 i$ site whose SOFs increase with pressures above the isostructural transition pressure. At the highest pressure, all the Si SOFs on the host sites $(16 i, 24 k$ and $6 c)$ converge to the same value.
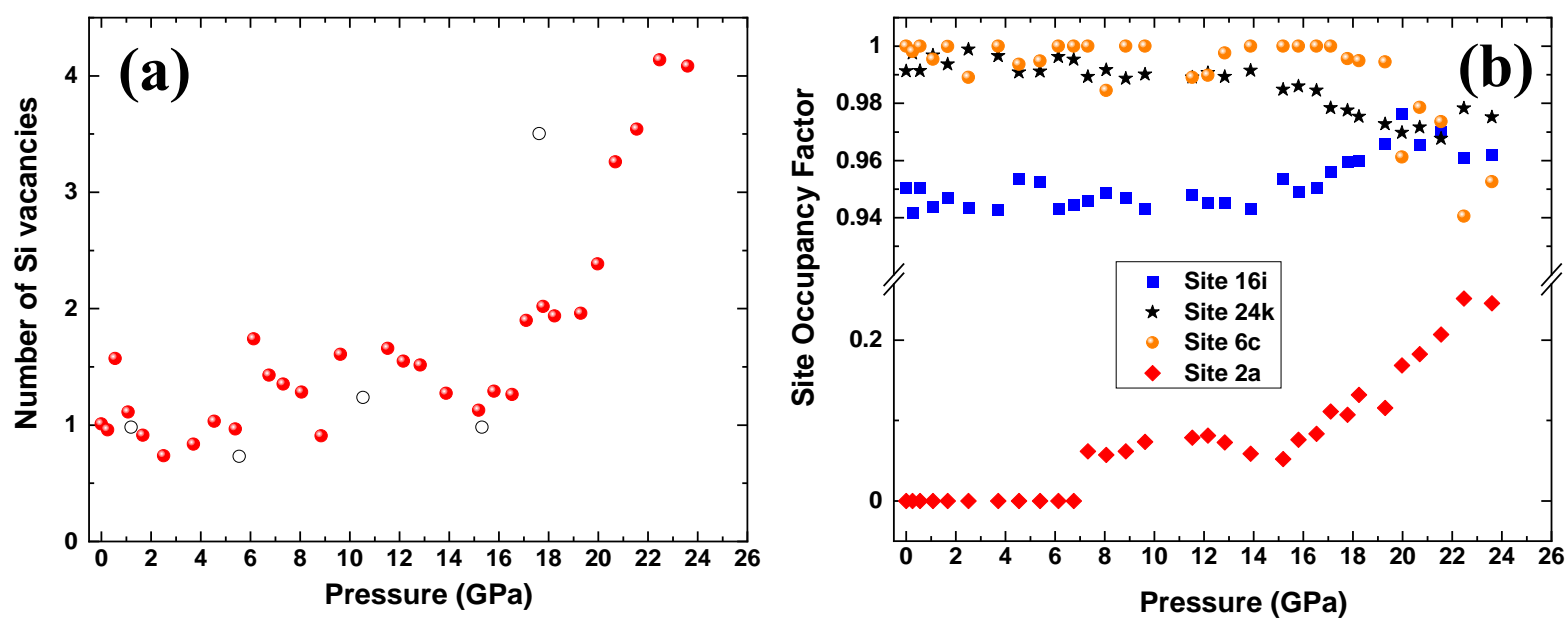

Figure 6 - (a) Refined number of Si vacancies as a function of pressure during the compression (full circles) and decompression (open circles), assuming the system is open (see text); (b) Refined Si Site Occupancy Factors (SOF) of the host 16i-, 24k- and $6 c$-sites and on the guest $2 a$-site as a function of pressure, in the hypothesis of a closed system (see text).

We refined the dataset of run 1 using both scenarios (Tab. SM-5). The goodness of fit is slightly in favor of scenario 2, with the hopping of $\mathrm{Si}$ in the vacant $\mathrm{Ba}(2 \mathrm{a})$ sites. Therefore, we tested scenario 2 by ab initio calculations based on density functional theory (DFT) under applied pressure. The fact that $a b$ initio DFT calculations have been found to accurately reproduce 
various experimental aspects of the lattice dynamics in $\mathrm{Ba}_{8} \mathrm{Si}_{46}[31,32,33]$ indicates that such an approach is well suited for this system.

Following the experimental observations, two types of model structures, with either a vacancy or a $\mathrm{Si}$ atom in one of the $\mathrm{Si}_{20}$ cages, were studied over a wide range of pressures (from 0 to 20 $\mathrm{GPa}$, in steps of $1.25 \mathrm{GPa})$ (Figure 7): $\mathrm{Ba}_{7} \mathrm{Si}_{46}\left(\mathrm{Ba}_{1} \square_{1} \mathrm{Ba}_{6} \mathrm{Si}_{46}\right)$ stands for a model structure with a vacancy on the $\mathrm{Ba}(2 a)$ site, $\mathrm{SiBa}_{7} \mathrm{Si}_{45}\left(\mathrm{Si}_{1} \mathrm{Ba}_{1} \mathrm{Ba}_{6} \mathrm{Si}_{45} \square_{1}\right)$ represents a structure for which a host Si (16i or $24 k$ ) atom has been placed at the vacant guest $2 a$-site, thus leaving a host vacancy. In Fig. 7a, the enthalpy difference between two vacancy containing model structures is depicted as a function of the pressure applied. Interestingly, at a pressure of about $8 \mathrm{GPa}$, the $\mathrm{SiBa}_{7} \mathrm{Si}_{45}$ compound becomes the most stable structure, which confirms the energy gain associated with the filling of the guest $2 a$-site by $\mathrm{Si}$. This pressure is in very good agreement with the start of filling of the vacant $2 a$ site observed experimentally. The delay in the effective transition (arising at $16.8 \mathrm{GPa}$ ) is probably due to the kinetics effects of the diffusion barrier a Si atom has to overcome to diffuse into an empty cage. The variation of transition pressure has already been observed depending on hydrostaticity and may also depend on the form of the crystals (powder $v s$ single crystal) [21].

Moreover, at $20 \mathrm{GPa}$, the formation of $\mathrm{SiBa}_{7} \mathrm{Si}_{45}$ is significantly more stable, by more than $\sim 1.7$ $\mathrm{eV}$, than a mixed phase of $\mathrm{Ba}_{7} \mathrm{Si}_{45}$ and the hexagonal $\mathrm{Si}(\mathrm{V})$ high-pressure phase, as proposed by Iitaka [27] (scenario 1) (see Tab. II). This mixed phase was found to be more stable than $\mathrm{Ba}_{7} \mathrm{Si}_{46}$ at $20 \mathrm{GPa}$ but with an enthalpy gain that was more than eight times lower than that corresponding to the $\mathrm{SiBa}_{7} \mathrm{Si}_{45}$ formation. This agrees perfectly with the lack of any signature of a secondary phase in the experimental diffraction data. Moreover, this enthalpy gain, together with the rather low barriers between the Si-sites at high pressure ( 0.1-0.3 eV at $20 \mathrm{GPa}$ [27]), promotes the diffusion of Si atoms, thereby explaining the leveling of the Si SOFs under pressure, as shown in Fig. 6b. It is worth noting that it is possible to place a framework vacancy on the $16 i$, the $24 k$ or the $6 c$ site. The $16 i$ and the $24 k$ site were found to be energetically almost identical, whereas a vacancy at the $6 c$ site was clearly less favorable at ambient pressure. It should be noted that the $\mathrm{Si}_{20}$ cage did not contain $\mathrm{Si}(6 \mathrm{c})$ atoms and hence the first step of the proposed transition should be the diffusion of a $\mathrm{Si}(16 i)$ or $\mathrm{Si}(24 k)$ atom to the cage center. 
Table II -Ab initio DFT calculation of the enthalpy formation of different vacancy model structures with the same amounts of $\mathrm{Ba}(7)$ and $\mathrm{Si}(46)$ atoms.

\begin{tabular}{cc}
\hline \hline Phase & Enthalpy at $20 \mathrm{GPa}$ in eV \\
\hline $\mathrm{Si}_{1} \mathrm{Ba}_{1} \mathrm{Ba}_{6} \mathrm{Si}_{6} \mathrm{Si}_{24} \mathrm{Si}_{15} \square_{1}\left(\mathrm{SiBa}_{7} \mathrm{Si}_{45}\right)$ & -144.476 \\
$\mathrm{Ba}_{1} \square_{1} \mathrm{Ba}_{6} \mathrm{Si}_{6} \mathrm{Si}_{24} \mathrm{Si}_{15} \square_{1}\left(\mathrm{Ba}_{7} \mathrm{Si}_{45}\right)+\mathrm{Si}(\mathrm{V})$ & -142.728 \\
$\mathrm{Ba}_{1} \square_{1} \mathrm{Ba}_{6} \mathrm{Si}_{46}$ & -142.556 \\
$\mathrm{Ba}_{2} \mathrm{Ba}_{5} \square_{1} \mathrm{Si}_{46}$ & -140.823 \\
\hline \hline
\end{tabular}

The pressure dependences of the unit cell volume calculated in $\mathrm{Ba}_{1} \square_{1} \mathrm{Ba}_{6} \mathrm{Si}_{46}$ and $\mathrm{SiBa}_{7} \mathrm{Si}_{45}$ are reported in Fig. $\mathbf{7 b}$. The volume of $\mathrm{Ba}_{1} \square_{1} \mathrm{Ba}_{6} \mathrm{Si}_{46}$ is larger than $\mathrm{SiBa}_{7} \mathrm{Si}_{45}$ over the whole pressure range. Thus, the transition from $\mathrm{Ba}_{1} \square_{1} \mathrm{Ba}_{6} \mathrm{Si}_{46}$ to $\mathrm{SiBa}_{7} \mathrm{Si}_{45}$ is concomitant with an abrupt decrease of the unit cell volume, as observed experimentally. In conclusion, the microscopic driving force of the transition corresponds to the filling of the empty 2a-sites by Si atoms.

(a)

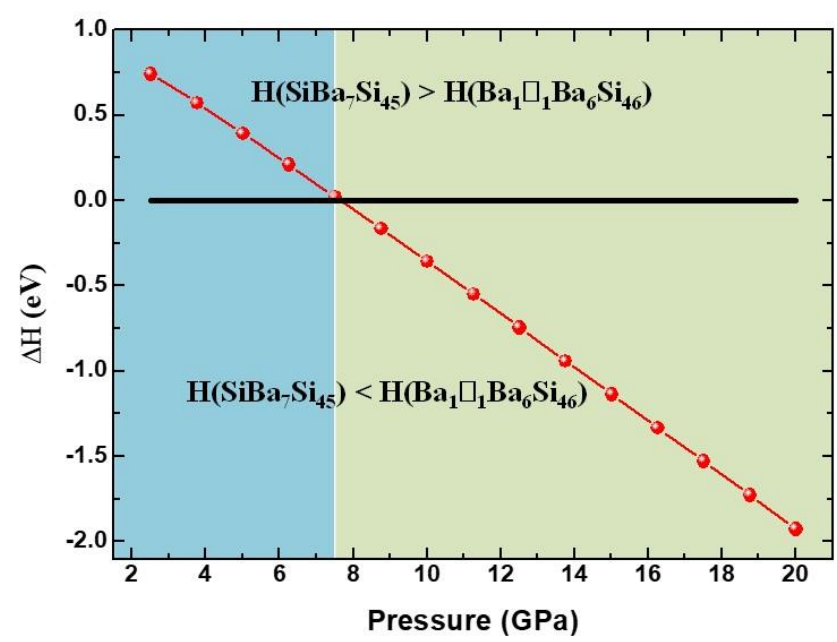

(b)

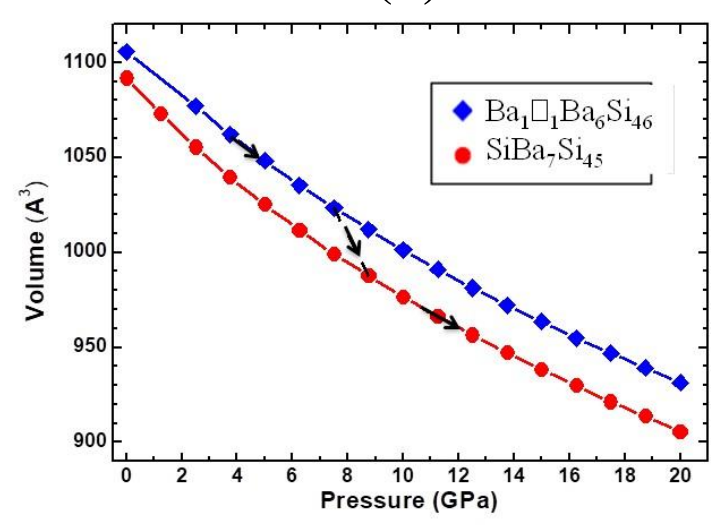

Figure 7. a) $A b$ initio determined enthalpy (H) difference of $\mathrm{H}\left(\mathrm{SiBa}_{7} \mathrm{Si}_{45}\right)-\mathrm{H}\left(\mathrm{Ba}_{1} \square_{1} \mathrm{Ba}_{6} \mathrm{Si}_{46}\right)$ up to a pressure of $20 \mathrm{GPa}$. Positive $\Delta \mathrm{H}$ indicates that the $\mathrm{Ba}_{1} \square_{1} \mathrm{Ba}_{6} \mathrm{Si}_{46}$ model is more stable, while negative $\Delta \mathrm{H}$ mean $\mathrm{SiBa}_{7} \mathrm{Si}_{45}$ is energetically favorable. b) Calculated unit cell volumes of $\mathrm{Ba}_{1} \square_{1} \mathrm{Ba}_{6} \mathrm{Si}_{46}$ (solid blue diamonds) and $\mathrm{SiBa}_{7} \mathrm{Si}_{45}$ (filled red circles) under pressure. 
In addition, in the framework of the "closed" scenario, the pressure dependence of the isotropic atomic displacement parameters $\left(\mathrm{U}_{\text {iso }}\right)$ for the guest and host atoms was refined and is reported in Fig. 8 for run 1. The $\mathrm{U}_{\text {iso }}$ values of the $\mathrm{Si}$ and $\mathrm{Ba}$ atoms increase abruptly as from the transition pressure. The observed stronger increase of $\mathrm{U}_{\text {iso }}(\mathrm{Ba}(2 a))$ can be understood as a consequence of the mixed $\mathrm{Ba} / \mathrm{Si}$ occupancy on the $2 a$ site and should be seen as $\mathrm{U}_{\mathrm{iso}}(\mathrm{Ba} / \mathrm{Si}(2 a))$. Indeed, the lighter $\mathrm{Si}(2 \mathrm{a})$ atom shows a much larger displacement amplitude. We confirm this statement by considering the atomic displacement parameters (ADPs) at $300 \mathrm{~K}$ obtained from ab initio DFT phonon calculations (see Method) for the $\mathrm{SiBa}_{7} \mathrm{Si}_{45}$ and the $\mathrm{Ba}_{1} \square_{1} \mathrm{Ba}_{6} \mathrm{Si}_{46}$ phase at a pressure of $10 \mathrm{GPa}$ (i.e., slightly above the theoretically determined transition pressure) (Table III). The $\mathrm{ADP}$ of the $\mathrm{Ba}(2 \mathrm{a})$ site is more than twice as high in $\mathrm{SiBa}_{7} \mathrm{Si}_{45}$, in good agreement with the experiment. On the other hand, the calculated ADPs associated with the other sites change relatively slightly, by about $5 \%$ in average. Thus, the experimental increase of the $\mathrm{Ba} \mathrm{U}_{\text {iso }}$ value at transition is in excellent agreement with $\mathrm{Si}$ moving into the $\mathrm{Si}_{20}$ cage.

Table III $-A b$ initio DFT calculations of the atomic displacement parameters $\left(\mathrm{U}_{\text {iso }}\right)$ obtained at $300 \mathrm{~K}$ and $10 \mathrm{GPa}$ in units of $10^{-4} \AA^{2}$.

\begin{tabular}{cccccc}
\hline \hline & $\mathrm{Si}(6 \mathrm{c})$ & $\mathrm{Si}(16 \mathrm{i})$ & $\mathrm{Si}(24 \mathrm{k})$ & $\mathrm{Ba}(2 \mathrm{a})$ & $\mathrm{Ba}(6 \mathrm{~d})$ \\
\hline $\mathrm{Ba}_{1} \square_{1} \mathrm{Ba}_{6} \mathrm{Si}_{46}$ & 8.318 & 8.243 & 8.592 & 6.312 & 10.339 \\
$\mathrm{SiBa}_{7} \mathrm{Si}_{6} \mathrm{Si}_{24} \mathrm{Si}_{15} \square_{1}$ & 7.973 & 8.374 & 8.066 & 14.24 & 9.403 \\
\hline \hline
\end{tabular}

The large increase of $U_{\text {iso }}$ with increasing pressure observed experimentally for all the guest and host atoms appears to be a side-effect at the transition due to the occurrence of atomic diffusion not considered in the simulation. It demonstrates that the isostructural transition originates from the pressure-induced activation of atomic diffusion, resulting in an entropy increase as already discussed. The opening of a new diffusion path for the $\mathrm{Si}$ atoms to the $\mathrm{Ba}$ free $2 a$ site acts as a catalyst. Intuitively, the higher the temperature, the lower the transition pressure will agree with the phase diagram shown in Fig. 2 d. 

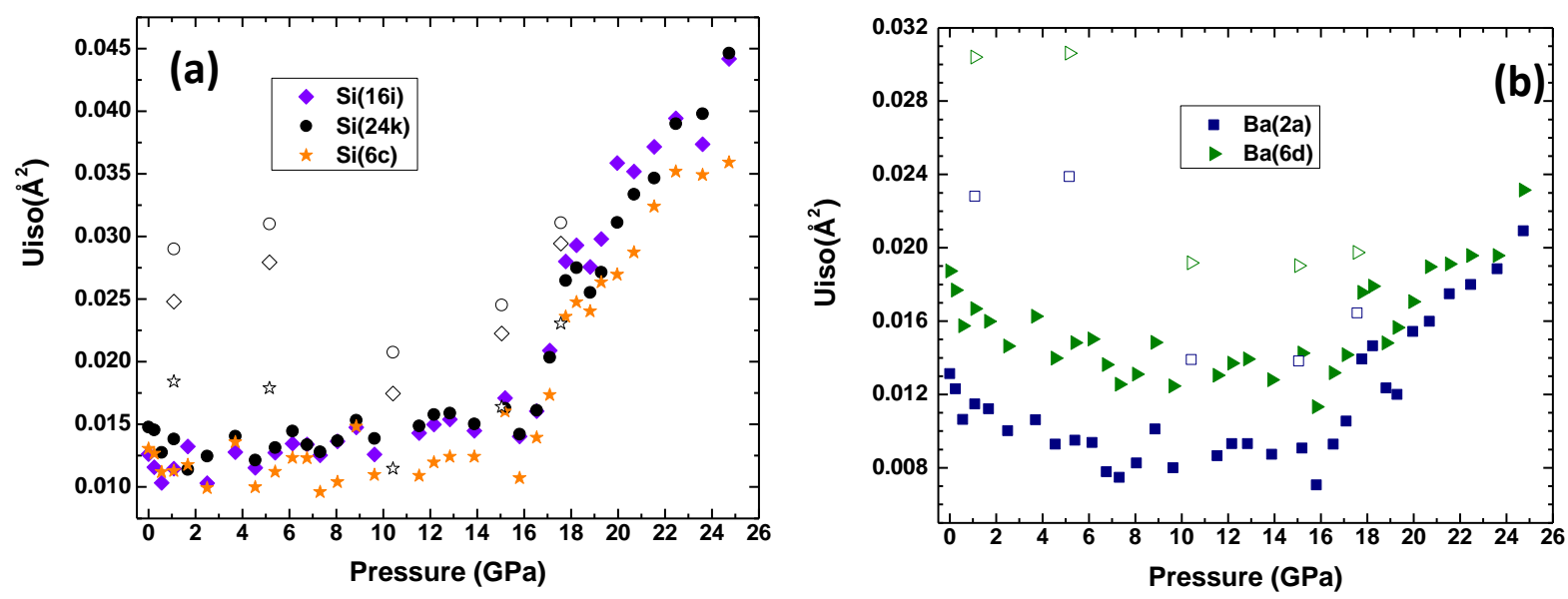

Figure 8 - Run 1 (300 K) isotropic atomic displacement parameters upon compression for (a) the silicon atoms at the $16 i$ (filled magenta diamond), $24 k$ (filled black diamond) and $6 c$ sites (filled yellow stars), (b) the Barium atoms at the $2 a$ (filled black square) and $6 d$ (filled green triangles) sites. In panels a) and b), the corresponding empty symbols describe the decompression.

Finally, the stability of a phase with some $\mathrm{Si}$ at the center of the cages also implies a drastic change of the electronic configuration of the Si atom having moved to the center of the dodecahedral cage, i.e. Si@Si 19 . We calculated the charge density distribution and the electronic density of states (eDOS) to investigate the changes in electronic structure (Fig. 9). It clearly appears that the $\mathrm{Si}$ atom in the cage center differs from the framework atoms. In particular, no $\mathrm{sp}^{3}$ like bonds from the cage center to the framework are observed, while at the same time additional electronic states, expected to be very localized, are observed close to the Fermi level. Moreover, charge density difference plots and Bader analysis indicate that the Si atom in the cage center is indeed charge neutral (Fig. 9). Thus, remarkably, the Si atom trap in the dodecahedral cage appears to be neutral and almost no deformation of its electronic clouds toward the induced vacancy on the cage is observed, while $\mathrm{Ba}$ atoms at the cluster center are known to donate their "s" electrons to the Si framework [45, 46]. 

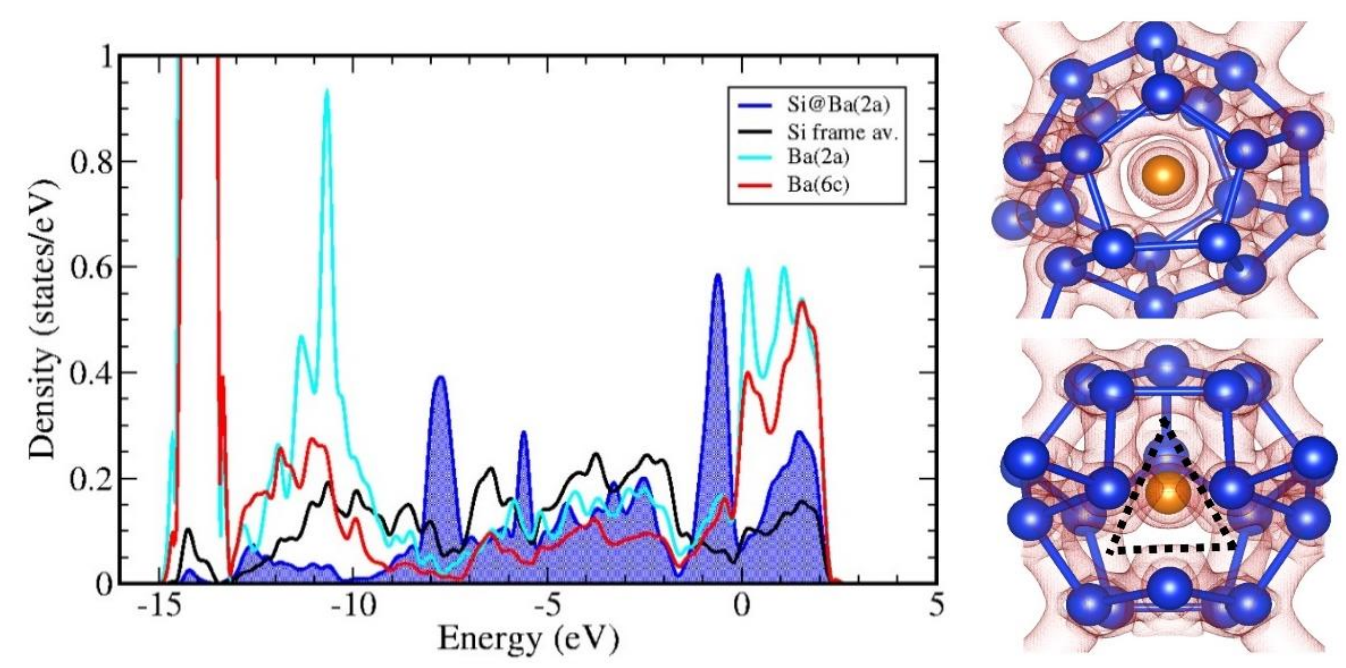

Figure 9: (color online) (a) Partial electronic density of states in $\mathrm{SiBa}_{7} \mathrm{Si}_{45}$ at $20 \mathrm{GPa}$. Si$\operatorname{host}(6 c, 16 i$ and $24 k)$ in black, $\operatorname{Si}(2 a)$ at the center of the dodecahedron in the blue shaded area, $\mathrm{Ba}(2 a)$ in cyan and $\mathrm{Ba}(6 d)$ in red. (b) Charge density 3D views of the defect $\mathrm{Si} @ \mathrm{Si}_{19}$ dodecahedral cage with a Si atom trap at the center and a vacancy on the cage

Assuming that the transition is isostructural, we finally propose to analyze it in the framework of the Landau theory of phase transitions [47]. In this case, it has been shown that the Landau potential is:

$$
L(\eta)=L_{0}+\frac{\alpha}{2} \cdot \eta^{2}+\frac{B}{3} \cdot \eta^{3}+\frac{\beta}{4} \cdot \eta^{4}
$$

This variational potential corresponds to the free energy only at the equilibrium value of the order parameter $\eta$ associated with the transition. The stability conditions for the crystalline state require that $\mathrm{B}<0$ and $\beta>0$ and the usual requirement on $\alpha$ is that it changes sign at the transition according to $\alpha=a\left(P-P_{c}\right)$, where $P_{C}$ is the pressure where phase I becomes unstable and $a$ is a constant.

The minimization of $L$ with respect to $\eta$ leads to two solutions:

$\eta=0$ which corresponds to the low-pressure phase $\mathrm{I}$,

$\eta=\frac{-B+\left(B^{2}+4 \alpha \beta\right)^{1 / 2}}{2 \beta}$ which corresponds to the high-pressure phase II.

This indicates that the order parameter varies as the square-root of $\mathrm{P}$ since the evolution of this quantity is expressed as $\eta=\frac{-B\left[\left(B^{2}-4 a \beta P_{C}\right)+4 a \beta P\right]^{1 / 2}}{2 \beta}$. 
As there is no symmetry breaking across the phase transition, $\eta$ should transform as the totally symmetric irreducible representation of the group. The order parameter corresponds to a physical quantity that changes spontaneously at the transition and which is null in phase I. A scalar such as the SOF of $\mathrm{Ba}(2 \mathrm{a})$ by $\mathrm{Si}$ atoms identified as the main features of the transition, corresponds to the symmetry requirement. In figure 10, the normalized SOF of site $2 \mathrm{a}$ by the $\mathrm{Si}$ atoms is plotted and shows a $\mathrm{P}^{1 / 2}$ variation, as expected. Note that, because of the constant filling of the 2a sites above $7 \mathrm{GPa}$ by $\mathrm{Si}$ atoms, this SOF value was taken as the reference for Phase I.

Moreover, experimentally, we observed a variation in the elastic properties across the transition, so an elastic contribution (secondary order parameter) including a coupling between the deformation and the primary order parameter should be included such that

$$
L(\eta, \varepsilon)=L_{0}+\frac{\alpha}{2} \cdot \eta^{2}+\frac{B}{3} \cdot \eta^{3}+\frac{\beta}{4} \cdot \eta^{4}+\lambda_{1} \varepsilon+\lambda_{2} \varepsilon^{2}+\delta \varepsilon \eta
$$

This is similar to the treatment done to account for the magnetostriction effect in the case of magnetic transition $[48,49]$ and considering that the spontaneous deformation is a consequence of the phase transition mechanism driven by the primary order parameter $\eta$.

The bilinear coupling in eq.(2) arises from the totally symmetric nature of the physical quantities (scalar) induced by the isostructural nature of the transition. Therefore, the volume change relative to the volume at ambient pressure, namely $\Delta \mathrm{V} / \mathrm{V}_{0}$, is a good candidate. Indeed, it changes across the transition and, being a scalar, it satisfies the no symmetry-breaking requirement associated with the isostructural character of the transition.

The equilibrium condition related to the deformation $\frac{\partial L}{\partial \varepsilon}=0$ yields

$$
\varepsilon=-\frac{\delta}{\lambda_{2}} \eta-\frac{\lambda_{1}}{\lambda_{2}}
$$

This relationship indicates that the deformation varies in the same fashion as the primary order parameter. The second term corresponds to the deformation not related to the transition, i.e. to the usual pressure-induced compression. The intrinsic volume change associated with the transition, i.e. the change of $\Delta \mathrm{V} / \mathrm{V}_{0}$ across the transition, or spontaneous deformation ( $\Delta_{\text {spontaneous}}$ ), is obtained by subtracting the compression contribution (unrelated to the phase transition) to the experimental variation $\Delta \mathrm{V} / \mathrm{V}_{0}$ under pressure (see Fig. SM-2 for details).

Figure 10 compares the primary order parameter with the spontaneous deformation and verified the linear relation between the filling of the $2 \mathrm{a}$ sites by $\mathrm{Si}$ atoms and the spontaneous variation of the relative volume. 


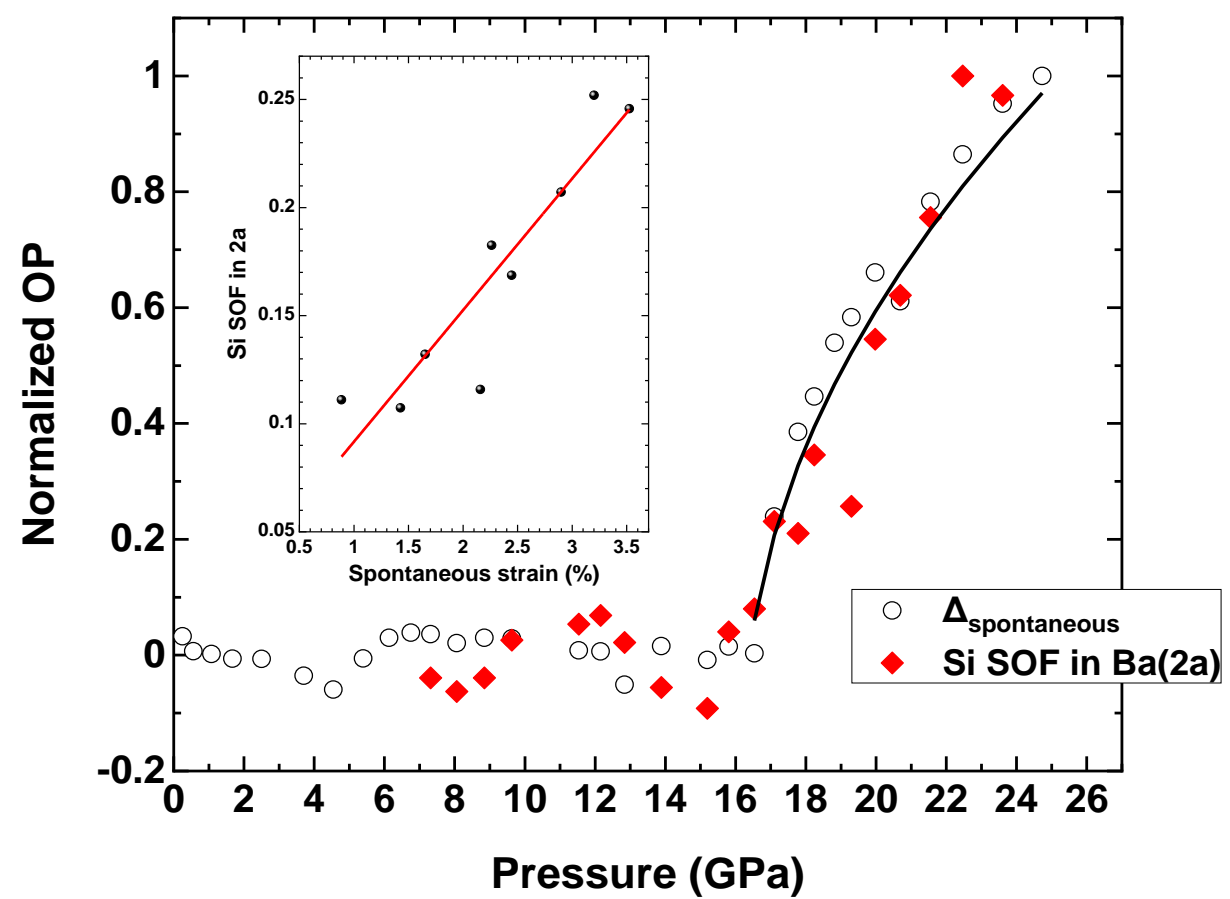

Figure 10 - Normalized number of $\mathrm{Si}$ atoms in the $2 \mathrm{a}$-site (red diamond) fitted with a $\mathrm{P}^{1 / 2}$ function, as expected by the analysis using the Landau theory of phase transition. This quantity is compared with $\Delta_{\text {spontaneous }}$ (open circle). The inset shows the linear relation between these two quantities above the transition, as expected from equation (3).

Structural refinement during the high-pressure experiment at low temperature (Run 2) cannot be achieved due to the degradation of crystal quality through the transition. Therefore, the SOF of the $\mathrm{Ba}(2 \mathrm{a})$ sites cannot be obtained. However, the pressure dependences of $\Delta_{\text {spontaneous }}$ at 180 and $300 \mathrm{~K}$ are reported in Fig. 11. The overall shape of the experimental $\Delta_{\text {spontaneous }}(\mathrm{P})$ is well reproduced by fits using order parameter expressions derived from the Landau theory (solid lines in Fig.11), indicating that the transition mechanism is similar. 


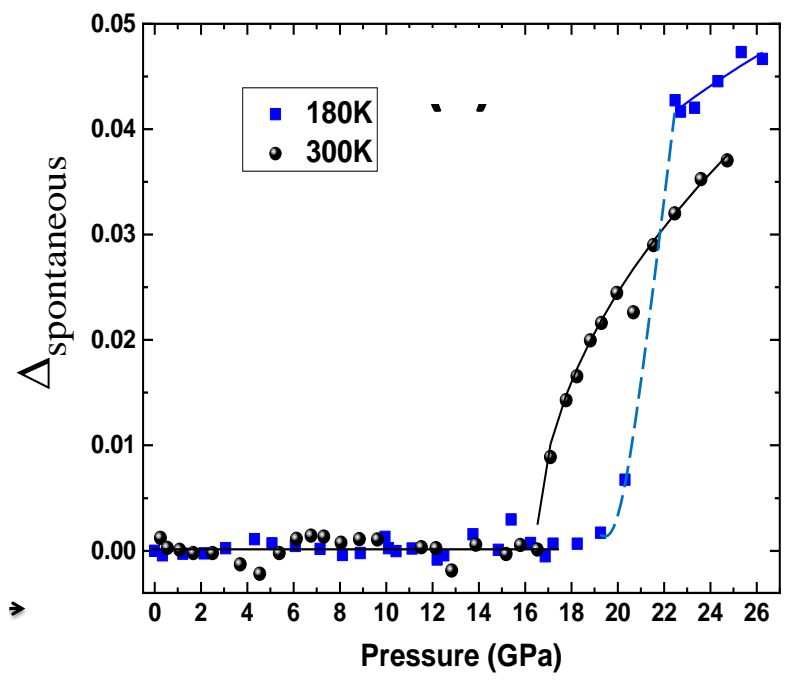

Figure $11-\Delta_{\text {spontaneous }}$ (see text) obtained as a function of pressure for runs 1 (black filled circles) and 2 (blue filled squares ). Solid lines are fits with the functional form derived from the Landau theory (see text).

\section{Conclusions}

Beyond the mechanism of the isostructural transition in of $\mathrm{Ba}_{1.5} \square_{0.5} \mathrm{Ba}_{6} \mathrm{Si}_{45} \square_{1}\left(\mathrm{Ba}_{7.5} \mathrm{Si}_{45}\right.$ ), this study illustrated a more general microscopic mechanism of isostructural phase transitions in complex crystalline intermetallic phases, based on a disorder-disorder transition among different isostructural point defect configurations proposed theoretically by Alavi [6]. Here the pressure acted as a reversible switch between two types of disorder which, without changing the average structure, impacted on the physical properties. The transition mechanism was associated with an order parameter that corresponded to the occurrence of a localized favored state of the Si atoms. This physical driving force was similar to the mechanism foreseen in the liquid-liquid transition (LLT) [50]. In this case as well, the transformation between liquid states was delimited by a first order phase transition line ended by a critical point. In the case of silicon, there is now a plethora of evidence of the existence of a liquid-liquid phase transformation that explains, for instance, the negative slope of the melting temperature with pressure or polyamorphism [50,51]. Okada et al. found evidence for the coexistence of two distinct bonding species, metallic and covalent, in the liquid state which supports the possibility of LLT [52]. These two states were also observed in our case and the occurence of metallic Si 
was the driving force of the transition, strengthening the relation between the LLT and the isostructural transformation observed in our experiments. Similarly, in Ce, it was shown that the extension of the liquid-liquid phase transition coincided with the well-known isostructural phase transitions in solid Ce induced by localized electronic transition [53]. This analogy should be explored in greater depth in the future to understand the relation between these isostructural phase transitions in solids and liquids. In addition, this mechanism of isostructural transitions based on the switching between defect configurations bridges the polymorphic phase transitions, i.e. the usual order-disorder transitions, and the polyamorphic ones, i.e. the amorphous-to-amorphous transitions. The latter are entropy-driven and correspond to a switch between different configurations of disorder in amorphous states [54]. Such transformations were observed in silicon clathrates [23].

As the transition is accompanied by the trapping of a silicon atom in a quasi-neutral configuration, this results in the occurrence of weakly coupled electronic states with energies close to the electrochemical potential, and makes the electronic density of states highly asymmetric on either side with a peak in the valence band. These states could correspond to resonant states which are known to be the source of high values of the Seebeck coefficient which appears squared in the expression of material thermoelectric efficiency $[55,56,57]$. Thus, this isostructural transition could point to a new strategy to boost the thermoelectric figure of merit in type-I clathrates and, more fundamentally, allow studying the effect of resonant states on thermoelectric transport properties. In the specific case of type-I silicon clathrate, which is superconductor for temperatures below $8 \mathrm{~K}$, changes in the electronic structure at the isostructural transition were found to be the origin of a sudden increase in the superconducting temperature [30]. This indicates that not only do the additional Si-weighted densities of states at the electrochemical potential contribute to electronic conductivity but that they also couple to phonons which might also participate in enhancing the contribution of phonon drag to the Seebeck coefficient. Thus, further studies on performing Seebeck measurements as a function of temperature across isostructural compounds would be very interesting.

\section{Acknowledgments}

The authors thank ESRF, Grenoble, for the beamtime allocation. R. Debord and S. Pailhès acknowledge support from the Lyon IDEX Scientific Breakthrough program for funding of the 
project IPPON. The authors would like to thank Olivier Perez (Crismat, EnsiCaen) for fruitful technical discussion on the analysis of the XRD data on complex crystals

\section{References}

1 K. Hoang and M.D. Johannes, Defect physics in complex energy materials, J. Phys.: Condens. Matter 30 (2018) 293001.

2 G. Jeffrey Snyder and E.S. Toberer, Complex thermoelectric materials, Nat. Mater. 7 (2008) 105.

3 E.S. Toberer, L.L. Baranowski, C. Dames, Advances in Thermal Conductivity, Ann. Rev. Mater. Res. 42 (2012) 179.

4 E.S. Toberer, A. Zevalkink, G.J. Snyder, Phonon engineering through crystal chemistry, J. Mater. Chem. 21 (2001) 15843.

5 Y. Grin, Inhomogeneity and anisotropy of chemical bonding and thermoelectric properties of materials, J. Solid State Chem. 274 (2019) 329.

6 A. Alavi, A.Y. Lozovoi, M.W. Finnis, Pressure-Induced Isostructural Phase Transition in AlRich NiAl Alloys, Phys. Rev. Lett. 83 (1999) 979.

7 J.M. Lawrence, P.S. Riseborough, R.D. Parks, Valence fluctuation phenomena, Rep. Prog. Phys. 44 (1981) 1.

8 K.T. Moore, L. Belhadi, F. Decremps, D.L. Farber, J.A. Bradley, F. Occelli, M. Gauthier, A. Polian, C.M. Aracne-Ruddle, Watching a metal collapse: examining cerium's $\gamma \leftrightarrow \alpha$ transformation using X-ray diffraction of compressed single and polycrystals, Acta Materialia 59 (2011) 6007.

9 N. Devaux, M. Casula, F. Decremps, S. Sorella, Electronic origin of the volume collapse in cerium, Phys. Rev. B 91 (2015) 081101.

10 S. Lupi, L. Baldassarre, B. Mansart et al. A microscopic view on the Mott transition in chromium-doped $\mathrm{V}_{2} \mathrm{O}_{3}$, Nat Commun. 1 (2010) 105. 
11 S. Banerjee, D. Nath, and B.K. Chaudhuri, Phenomenological theory of the isostructural phase transition in $\mathrm{H}$-bonded $\mathrm{SnCl}_{2}\left(\mathrm{H}_{2} \mathrm{O}\right)_{2}$ and $\mathrm{SnCl}_{2} \cdot\left(\mathrm{H}_{2} \mathrm{O}\right)_{\mathrm{x}}\left(\mathrm{D}_{2} \mathrm{O}\right)_{2-\mathrm{x}}$ with a pseudospin model, Phys. Rev. B 25 (1982) 1883.

$12 \mathrm{Y}$. Ishibashi, An Interpretation of the Isomorphous Transition in $\mathrm{SnCl}_{2} \cdot 2 \mathrm{H}_{2} \mathrm{O}$, J. Phys. Soc. Jpn. 49 (1980) 619.

13 Z. Hiroi, J.-i. Yamaura, and K. Hattori, Rattling Good Superconductor: $\beta$-Pyrochlore Oxides $\mathrm{AOs}_{2} \mathrm{O}_{6}$, J. Phys. Soc. Jpn. 81 (2012) 011012.

14 T. Takabatake, K. Suekuni, T. Nakayama, E. Kaneshita, Phonon-glass electron-crystal thermoelectric clathrates: Experiments and theory, Rev. Mod. Phys. 86 (2014) 669.

15 G. S. Nolas, editor, The Physics and Chemistry of Inorganic Clathrates, Vol. 199, Springer Series in Materials Science (Springer, Heidelberg, 2015).

16 J.S. Tse, S. Desgreniers, Z.Q. Li, M.R. Ferguson, Y. Kawazoe, Structural Stability and Phase Transitions in $\mathrm{K}_{8} \mathrm{Si}_{46}$ Clathrate under High Pressure, Phys. Rev. Lett. 89 (2002) 195507.

17 A. San-Miguel, P. Mélinon, D. Connétable, X. Blase, F. Tournus, E. Reny, S. Yamanaka, J.-P. Itié, Pressure stability and low compressibility of intercalated cagelike materials: The case of silicon clathrates, Phys. Rev. B 65 (2002) 054109.

18 T. Kume, H. Fukoaka, T. Koda, S. Sasaki, H. Shimizu, S. Yamanaka, High-Pressure Raman Study of Ba Doped Silicon Clathrate, Phys. Rev. Lett. 90 (2003) 155503.

19 L. Yang, Y.M. Ma, T. Iitaka, J.S. Tse, K. Stahl, Y. Ohishi, Y. Wang, R.W. Zhang, J.F. Liu, H.-K. Mao, J.Z. Jiang, Pressure-induced phase transformations in the $\mathrm{Ba}_{8} \mathrm{Si}_{46}$ clathrate, Phys. Rev. B 74 (2006) 245209.

20 J.S. Tse, L. Yang, S.J. Zhang, C.Q. Jin, Ch. J. Sahle, C. Sternemann, A. Nyrow, V. Giordano, J. Z. Jiang, S. Yamanaka, S. Desgreniers, C.A. Tulk, Pressure-induced electron topological transitions in Ba-doped Si clathrate, Phys. Rev B 84 (2011) 184105.

21 A San-Miguel and P Toulemonde, High-pressure properties of group IV clathrates, High Pressure Research 25 (2005) 159.

22 H. Shimizu, T. Kume, T. Kuroda, S. Sasaki, H. Fukuoka, S. Yamanaka, High-pressure Raman study of the iodine-doped silicon clathrate $\mathrm{I}_{8} \mathrm{Si}_{44} \mathrm{I}_{2}$, Phys. Rev. B 68 (2003) 212102. 
23 D. Machon, P. Toulemonde, P.F. McMillan, M. Amboage, A. Muñoz, P. RodríguezHernández, A. San-Miguel, High-pressure phase transformations, pressure-induced amorphization, and polyamorphic transition of the clathrate $\mathrm{Rb}_{6.15} \mathrm{Si}_{46}$, Phys. Rev. B 79 (2009) 184101.

24 A. San-Miguel, A. Merlen, P. Toulemonde, T. Kume, S. LeFloch, A. Aouizerat, S. Pascarelli, G. Aquilanti, O. Mathon, T. LeBihan, J.P. Itié, S. Yamanaka, Pressure-induced homothetic volume collapse in silicon clathrates, Europhys. Lett. 69 (2005) 556.

25 J.S. Tse, R. Flacau, S. Desgreniers, T. Iitaka, J.Z. Jiang, Electron density topology of highpressure $\mathrm{Ba}_{8} \mathrm{Si}_{46}$ from a combined Rietveld and maximum-entropy analysis, Phys. Rev. B 76 (2007) 174109.

26 T. Kume, T. Koda, S. Sasaki, H. Shimizu, J.S. Tse, High-pressure Raman study of the potassium-doped silicon clathrate $\mathrm{K}_{8} \mathrm{Si}_{46}$, Phys. Rev. B 70 (2004) 052101.

27 T. Iitaka, Pressure-induced isostructural phase transition of metal-doped silicon clathrates, Phys. Rev. B 75 (2007) 012106.

28 H. Fukuoka, J. Kiyoto, S. Yamanaka, Superconductivity of Metal Deficient Silicon Clathrate Compounds, Ba8-x $\mathrm{Si}_{46}(0<\mathrm{x} \leq 1.4)$, Inorg.Chem. 42 (2003) 2933.

29 R. Castillo, W. Schnelle, M. Bobnar, U. Burkhardt, B. Böhme, M. Baitinger, U. Schwarz, Y. Grin, The Clathrate $\mathrm{Ba}_{8-\mathrm{x}} \mathrm{Si}_{46}$ Revisited: Preparation Routes, Electrical and Thermal Transport Properties, Z. Anorg. Allg. Chem. 641 (2015) 206.

30 F. Morales, M. Núñnez-Regueiro, P. Toulemonde, D. Machon, S. Le Floch, V. Pischedda, P. Lagarde, A.-M. Flank, J. P. Itié, A. San-Miguel, Enhanced high-pressure superconductivity and local structure of the $\mathrm{Ba}_{8} \mathrm{Si}_{46}$ clathrate, Phys. Rev. B 94 (2016) 104507.

31 H. Euchner, S. Pailhès, V. M. Giordano, M. de Boissieu. Understanding lattice thermal conductivity in thermoelectric clathrates: A density functional theory study on binary Si-based type-I clathrates, Phys. Rev. B 97 (2018) 014304.

32 S. Pailhès, H. Euchner, V. M. Giordano, R. Debord, A. Assy, S. Gomès, A. Bosak, D. Machon, S. Paschen, M. de Boissieu, Localization of Propagative Phonons in a Perfectly Crystalline Solid, Phys. Rev. Lett. 113 (2014) 025506. 
33 R. Viennois, M. M. Koza, R. Debord, P. Toulemonde, H. Mutka, S. Pailhès, Anisotropic low-energy vibrational modes as an effect of cage geometry in the binary barium silicon clathrate Ba ${ }_{24} \mathrm{Si}_{100}$, Phys. Rev. B 101 (2020) 224302.

34 F. Datchi, A. Dewaele, P. Loubeyre, R. Letoullec, Y. Le Godec, B. Canny, Optical pressure sensors for high-pressure-high-temperature studies in a diamond anvil cell, High Press. Res., 27 (2007) 447.

35 M. Merlini and M. Hanfland, Single-crystal diffraction at megabar conditions by synchrotron radiation, High Press. Res. 33 (2013) 511.

36 G. Kresse and J. Furthmüller, Efficient iterative schemes for ab initio total-energy calculations using a plane-wave basis set, Phys. Rev. B 54 (1996) 11169-11186.

37 G. Kresse and D Joubert, From ultrasoft pseudopotentials to the projector augmented-wave method, Phys. Rev. B 59 (1999) 1758-1775.

38 Y. Wang and J. Perdew, Accurate and simple analytic representation of the electron-gas correlation energy, Phys. Rev. B 45 (1992) 13244.

39 K. Parlinski, software PHONON, 2005

40 D. Connétable, First-principles calculations of carbon clathrates: Comparison to silicon and germanium clathrates, Phys. Rev. B 82 (2010) 075209.

41 R.J. Angel, Equations of state. Reviews in Mineralogy and Geochemistry 41 (2000) 35-59.

42 Y. Liang, B. Böhme, L. Vasylechko, M. Baitinger, Y. Grin, In-situ investigation of the thermal decomposition of clathrate-I Ba ${ }_{6.2} \mathrm{Si}_{46}$, J. Phys. Chem. Solids 74 (2013) 225.

43 R. Lortz, R. Viennois, A. Petrovic, Y. Wang, P. Toulemonde, C. Meingast, M. Marek Koza, H. Mutka, A. Bossak, A. San Miguel, Phonon density of states, anharmonicity, electron-phonon coupling, and possible multigap superconductivity in the clathrate superconductors $\mathrm{Ba}_{8} \mathrm{Si}_{46}$ and $\mathrm{Ba}_{24} \mathrm{Si}_{100}$ : Factors behind large difference in Tc, Phys. Rev. B 77 (2008) 224507.

$44 \mathrm{~J}$. Garai and A. Laugier, The temperature dependence of the isothermal bulk modulus at 1 bar pressure, J. Appl. Phys. 101 (2007) 023514. 
45 T.F.T. Cerqueira, S. Pailhès, R. Debord, V.M. Giordano, R. Viennois, J. Shi, S. Botti, M.A.L. Marques, Prediction and Synthesis of a Non-Zintl Silicon Clathrate, Chem. Mater. 28 (2016) 3711.

46 N. Kamakura, T. Nakano, Y. Ikemoto, M. Usuda, H. Fukuoka, S. Yamanaka, S. Shin, K. Kobayashi, Role of Ag doping in $\mathrm{Ba}_{8} \mathrm{Si}_{46}$ compounds, Phys. Rev. B 72 (2005) 014511.

47 P. Tolédano and V. Dmitriev, Reconstructive Phase Transitions (World Scientific, Singapore, 1996).

48 P. Tolédano, H. Katzke, D. Machon, J. Phys.: Cond. Matter 46 (2010) 466002.

49 P. Tolédano and J.C. Tolédano, Landau Theory Of Phase Transitions, The: Application To Structural, Incommensurate, Magnetic And Liquid Crystal Systems (World Scientific Publishing Company, 1987).

50 H. Tanaka, Liquid-liquid transition and polyamorphism, J. Chem. Phys. 153 (2020) 130901.

51 P. F McMillan, M. Wilson, D. Daisenberger, D. Machon, A density-driven phase transition between semiconducting and metallic polyamorphs of silicon, Nat. Mater. 4 (2005) 680.

52 J. T. Okada, P. H.-L. Sit, Y. Watanabe, Y. J. Wang, B. Barbiellini, T. Ishikawa, M. Itou, Y. Sakurai, A. Bansil, R. Ishikawa, M. Hamaishi, T. Masaki, P.-F. Paradis, K. Kimura, T. Ishikawa, S. Nanao, Persistence of Covalent Bonding in Liquid Silicon Probed by Inelastic XRay Scattering, Phys. Rev. Lett. 108 (2012) 067402.

53 A. Cadien, Q.Y. Hu, Y. Meng, Y. Q. Cheng, M.W. Chen, J. F. Shu, H. K. Mao, H.W. Sheng, First-Order Liquid-Liquid Phase Transition in Cerium, Phys. Rev. Lett. 110 (2013) 125503.

54 D. Machon, F. Meersman, M.C. Wilding, M. Wilson, P.F. McMillan, Pressure-induced amorphization and polyamorphism: Inorganic and biochemical systems, Progr. Mater. Sc. 61 (2014) 216.

55 J. P. Heremans, B. Wiendlocha, A. M. Chamoire, Resonant levels in bulk thermoelectric semiconductors, Energy Environ. Sci. 5 (2012) 5510-5530.

56 B. Wiendlocha, Thermopower of thermoelectric materials with resonant levels: PbTe:Tl versus PbTe:Na and $\mathrm{Cu}_{1-\mathrm{x}} \mathrm{Ni}_{\mathrm{x}}$, Phys. Rev. B 97 (2018) 205203. 
57 S. Thébaud, Ch. Adessi, S. Pailhès, G. Bouzerar, Boosting the power factor with resonant states: A model study, Phys. Rev. B 96 (2017) 075201. 